\title{
Water masers in the star forming region in L1287
}

\author{
Hiroshi Imai* \\ Department of Physics, Faculty of Science, Kagoshima University, Japan \\ E-mail: hiroimaiesci.kagoshima-u.ac.jp
}

\section{Huib Jan van Langevelde}

Joint Institute for VLBI in Europe, the Netherlands

Sterrewacht Leiden, Leiden University, the Netherlands

E-mail: langevelde@ jive.nl

\section{Tomofumi Umemoto}

Nobeyama Radio Observatory, National Astronomical Observatory, Japan

E-mail: tomofumi.umemoto@nao.ac.jp

\begin{abstract}
Using the EVN, we have observed water maser emission in the star forming region in L1287. It has been suggested that the maser emission is associated with an infalling-rotating disk around a young stellar object with the mass of $3 M_{\odot}$. The distribution of maser radial velocities has persisted since previous VLA observations, supporting the above interpretation. Unfortunately, maser feature proper motions were not reliably traced because of the rapid temporal variation of maser feature locations between the observation epochs. We also found new maser features located in the SSW direction with respect to the known maser feature cluster, or in the same direction as that of the large-scale $\mathrm{CO}$ outflow. It also indirectly supports the existence of a disk/outflow system. However, it is also possible that all detected maser features are associated with an outflow with an extremely wide opening angle or an equatorial outflow.
\end{abstract}

The 8th European VLBI Network Symposium

September 26-29, 2006

Toruń, Poland

${ }^{*}$ Speaker. 


\section{Introduction}

Direct detection of mass accretion onto a young stellar object (YSO) and its surrounding disk should be the most important observational challenges for elucidating the star formation process. The mass accretion process increases stellar mass and, together with the outflow, determines its final value. Angular resolution achieved by VLBI ( $<1$ mas) makes it possible to directly detect the mass accretion. Water maser sources should be the possible targets for this purpose. We have known three candidates of the water maser study: L1287 (Fiebig 1997 and references therein), IRAS 16293-2422 [4], G192.16-3.84 ([5]; [3] and references therein), and other few targets. One of the final goals of the water maser study may be to detect maser proper motions for unambiguously finding out three-dimensional spatio-kinematics of YSO disks.

Here, we report multi-epoch EVN observations of the L1287 water masers. At two of the observation epochs, we detected several water maser features in L1287. We are also attempting the phase-referencing technique using data of the reference source GT 0032+612 ( 100 mJy) to more precisely estimate the absolute coordinates of the maser features and the secular motion of the whole L1287 maser source. Such high-precision astrometry using extragalactic QSO as position reference is also required because we are able to precisely superpose multi-epoch maser maps in a common coordinate system and to more accurately detect the maser kinematics.

\section{Results and discussion}

The radial velocity distribution found in the EVN observations is well elucidated by superposing two maser maps obtained at two observation epochs using the brightest maser feature as a position reference (see figure 1). The reference feature and other few features are located in the similar relative positions and believed to survive between the two observation epochs. The revealed velocity distribution is well consistent with that found in previous VLA observations (Fiebig 1997) and explained by a model of a rotating-infalling gas disk. The maser emission may be excited by collision of gas clumps impinging onto the disk with the disk gas at the disk mid-plane where sufficiently high gas density and temperature are found.

Because the epochs of observations leading to detection of many features were separated by 17 months, most of maser features quenched or were newly born between the two epochs. In such circumstances, unfortunately, it was difficult to trace maser feature proper motions. Only two maser features, including the reference feature, show stable relative positions, but meaningful maser proper motions could not be measured. Therefore, we have not yet obtained direct evidence for the existence of a YSO disk.

On the other hand, we detected new maser features that are located at $(-132,-426)$ [mas], in the SSW direction from the known feature cluster and associated with the CO outflow found by Yang et al. [6]. The feature locations have an angle offset of $\sim 25^{\circ}$ from the disk rotation/outflow axis and the major $\mathrm{CO}$ outflow axis. This angle gives us a lower limit of an estimated outflow opening angle, or a cavity of the inflow of gas clumps. The trajectory plane of impinging clumps passing the feature location reaches to the closest point of clump impinging on the disk $r \sim 10$ mas from the central YSO. This would allow us to study gas kinematics in very vicinity of the central YSO, which includes mass accretion onto the disk. However, it remains that all detected maser 


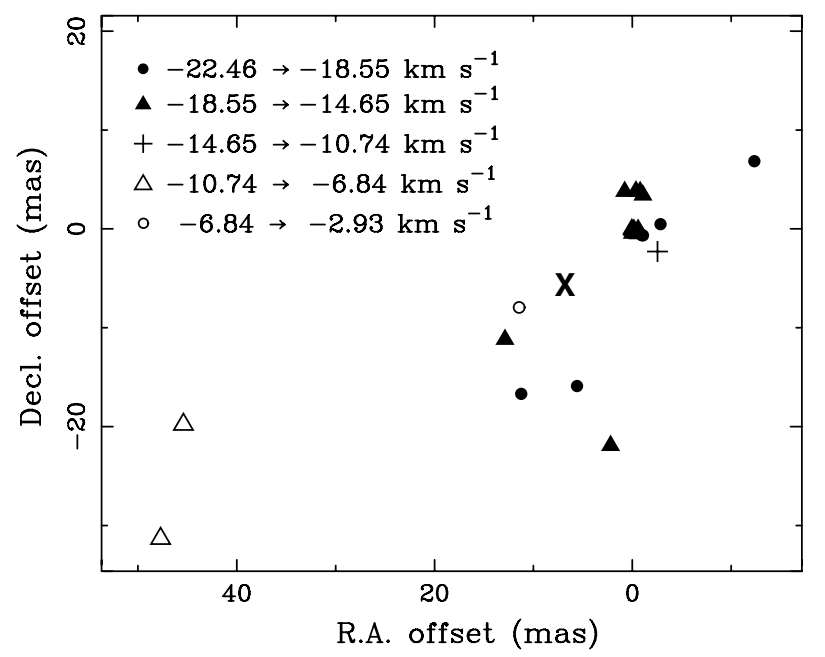

Figure 1: Maser distribution found by superposition of those found in June 2003 and November 2004. The position reference feature is located at the map origin. A cross symbol indicates the possible dynamical centre of the gas disk proposed by Fiebig [1].

features are associated with an outflow with an extremely wide opening angle or an equatorial outflow (e.g. [2]).

\section{Acknowledgments}

The EVN is a joint facility of European, Chinese, South African and other radio astronomy institutes funded by their national research councils. We are very grateful to Bob Campbell and Andreas Brunthaler, EVN friends, for the project support and Ricardo Cessaroni for important comments. H.I. has been financially supported by Grant-in-Aid for Scientific Research from Japan Society for Promotion Science (18740109).

\section{References}

[1] D. Fiebig, L $1287\left(\mathrm{H}_{2} \mathrm{O}\right)$ : accretion disk-impinging-clumps traced by masers?, A\&Ap 327, 758 (1997)

[2] C. Goddi \& L. Moscadelli, Tracing the base of protostellar wind(s) towards the high-mass star forming region AFGL 5142: VLA continuum and VLBA $\mathrm{H}_{2} 2 \mathrm{O}$ maser observations, A\&Ap 447, 577 (2006)

[3] H. Imai, T. Omodaka, T. Hirota, T. Umemoto, K. Sorai \& T. Kondo, A collimated jet and an infalling-rotating disk in G192.16-3.84 traced by $\mathrm{H}_{2} \mathrm{O}$ maser emission, PASJ 58, 883 (2006)

[4] H. Imai, T. Iwata \& M. Miyoshi, Rotation-Infall Motion around the Protostar IRAS 16293-2422 Traced by Water Maser Emission, PASJ 51473 (1999)

[5] D. Shepherd, T. Borders, M. Claussen, Y. Shirley \& S. Kurtz, The Circumstellar Environment of the Early B Protostar G192.16-3.84 and the Discovery of a Low-Mass, Protostellar Core, ApJ 614, 211 (2004)

[6] J. Yang, T. Umemoto, T. Iwata \& Y. Fukui, A millimeter-wave line study of L1287 - A case of induced star formation by stellar wind compression?, ApJ 455, 175 (1991) 